\title{
Virtual Reality Games for People Using Wheelchairs
}

\author{
Kathrin Gerling \\ KU Leuven, Department of \\ Computer Science \\ Leuven, Belgium \\ kathrin.gerling@kuleuven.be
}

\author{
Liam Mason \\ University of Lincoln, School \\ of Computer Science \\ Lincoln, UK \\ lmason@lincoln.ac.uk
}

\author{
Patrick Dickinson \\ University of Lincoln, School \\ of Computer Science \\ Lincoln, UK \\ pdickinson@lincoln.ac.uk
}

\author{
Adalberto L. Simeone \\ KU Leuven, Department of \\ Computer Science \\ Leuven, Belgium \\ adalberto.simeone@kuleuven.be
}

\author{
Kieran Hicks \\ University of Lincoln, School \\ of Computer Science \\ Lincoln, UK \\ khicks@lincoln.ac.uk
}

\begin{abstract}
Virtual Reality (VR) holds the promise of providing engaging embodied experiences, but little is known about how people with disabilities engage with it. We explore challenges and opportunities of VR gaming for wheelchair users. First, we present findings from a survey that received 25 responses and gives insights into wheelchair users' motives to (non-) engage with VR and their experiences. Drawing from this survey, we derive design implications which we tested through implementation and qualitative evaluation of three full-body VR game prototypes with 18 participants. Our results show that VR gaming engages wheelchair users, though nuanced consideration is required for the design of embodied immersive experiences for minority bodies, and we illustrate how designers can create meaningful, positive experiences.
\end{abstract}

\section{Author Keywords}

Accessibility, games, virtual reality.

\section{CCS Concepts}

-Applied computing $\rightarrow$ Computer games; Please use the 2012 Classifiers and see this link to embed them in the text: https://dl.acm.org/ccs/ccs_flat.cfm

\section{INTRODUCTION}

Virtual Reality (VR) environments in general and VR games in particular have become increasingly popular. However, the current state of art exhibits little consideration of accessibility concerns. While anecdotal evidence of access barriers in games (e.g. [6]), and an industry survey on disability and VR [1]) point to systemic issues, academia only slowly addresses questions on the accessibility of modern VR systems.

\footnotetext{
Permission to make digital or hard copies of all or part of this work for personal or classroom use is granted without fee provided that copies are not made or distributed for profit or commercial advantage and that copies bear this notice and the full citation on the first page. Copyrights for components of this work owned by others than the author(s) must be honored. Abstracting with credit is permitted. To copy otherwise, or republish, to post on servers or to redistribute to lists, requires prior specific permission and/or a fee. Request permissions from permissions@ acm.org.

CHI'20, April 25-30, 2020, Honolulu, HI, USA

(C) 2020 Copyright held by the owner/author(s). Publication rights licensed to ACM. ISBN 978-1-4503-6708-0/20/04 ..\$15.00
}

DOI: https : //doi .org/10.1145/3313831.3376265
While recent explorations consider VR gaming for players with visual impairment ${ }^{1}$ [31] and playful VR environments for neurodiverse children [7], the body- and movement-centric control scheme of many VR environments can be challenging for individuals with mobility impairment. They often combine hand-held controls with locomotion and/or extensive upper body movement which are not well situated to the movement of wheelchair users.

We address this issue through the design and development of a set of VR games functioning as experience prototypes, which allow us to explore varied preferences and needs of wheelchair users [32]). Our research followed a three-step process: First, we surveyed perspectives of wheelchair users of VR gaming. Responses from 25 participants show general interest in VR gaming and generally exploring novel technologies, but reveal a wide range of access barriers, including a need for adaptive control schemes and nuanced perspectives on disability representation. Second, we build on these results and existing work on wheelchair game controls to develop three accessible movement-based VR game prototypes to gain contextualised insights into the play experience of wheelchair users: Karamaisu Slope is a wheelchair-controlled skiing simulation, Dungeon Spell is a rail shooter which can be controlled through upper-body movement, and Space Travel is a first-person endless runner set in space using head movements as input. Results of two related user studies with a total of 18 participants show that VR games are generally engaging for wheelchair users. However, we also found that nuanced consideration is required when designing adaptive interaction paradigms or introducing partially automated gameplay. Drawing from these findings, we identify considerations for the design of adaptive and flexible body-based VR game interaction for wheelchair users, and reflect on and disability representation in VR.

We augment existing research by a structured exploration of wheelchair use and VR games, combining survey-based

\footnotetext{
${ }^{1}$ Our paper uses a mix of identity-first and person-first language. Different groups of people and different cultures appreciate different terminology.
} 
research with technological exploration. Additionally, we identify transferable implications for the design of wheelchairaccessible VR games. Finally, we provide a foundation for further discussion of wider challenges and opportunities for players with disabilities in VR.

VR carries the potential of creating immersive experiences for entertainment and beyond, and designers need to appropriately account for the situated engagement of marginalized groups with VR technology. Our work offers insights into how players with minority bodies engage with VR games with the intent to guide inclusive design processes.

\section{RELATED WORK}

Our research draws on previous work on the accessibility of VR environments for users with disabilities and wheelchairaccessible movement-based games.

\section{Accessibility of Virtual Reality for Disabled People}

Most innovation on wheelchair accessibility in VR occurs within community-driven projects. For example, WalkinVR [4] maps movement in SteamVR [3] onto hand-held controls, which removes the requirement for locomotion. While the system hugely improves overall accessibility of any VR software published on Steam [2], it also reduces the degree of full-body physical involvement in the virtual environment.

Several research projects explore the potential of VR for therapy and rehabilitation for individuals with disabilities through applied research, for example, learning environments for children with cognitive impairment [27], VR systems for stroke rehabilitation [21], or the potential of VR in vocational rehabilitation [8]). However, little academic work specifically explored general accessibility of VR as is common in other areas of Human-Computer Interaction (e.g., in a survey of game accessibility challenges [33]).

Within industry, a survey suggests that sight lines need to be adapted to account for seating height, and that some actions, such as reaching for items, require refinement [17]. Even though their survey in collaboration with the Disability Visibility Project [1] reveals that physical actions such as crouching, balancing, and moving are the most challenging activities to perform, they do not further address the issue of locomotion in VR.

\section{Wheelchair-controlled Playful Systems and Games}

Previous explorations of wheelchair-accessible game design focus on game control based on powered wheelchair interfaces or integrate physical effort and real-world wheelchair movement. GAMEWheels [13] leverages a mechanical wheelchair mount for game input, allowing players to control games through propulsion in moderate-to-vigorous exercise [25]. Building on off-the-shelf gaming hardware, AstroWheelie [12] uses accelerometry to detect wheelchair movement in a space-themed game. KINECTWheels [15] is a toolkit that supports the design of wheelchair-controlled games through camera-based player tracking - as evidenced by a system for older adults [16]. An extension for powered wheelchairs [14] effectively engages wheelchair users in physical play. Leveraging a different angle on camera-based player tracking, iGYM
[18] detects large-scale player movement from above and projects a playing field onto the floor, inviting both disabled and non-disabled players to engage with each other. Powered wheelchairs have also been used for game input without direct physical movement: Wheelchair-Rift [20] integrates similar joystick controls in a VR environment. However, the visual design of the system is simplistic, lacks central engaging elements of VR games, and the game was not evaluated with powered wheelchair users. Hence, little is known about wheelchair-accessible controls for playful inaction in VR or the specific design challenges for VR games for wheelchair users.

We address this issue from three perspectives: (1) a survey on wheelchair users' perspectives on VR systems, (2) three case studies of wheelchair-accessible VR games, (3) an evaluation of these games with wheelchair users. Reflecting on these results, we provide design implications for wheelchairaccessible full-body VR games.

\section{PART 1: SURVEYING WHEELCHAIR USERS' PERSPEC- TIVES ON VR GAMING}

We developed an online survey distributed via social media channels to explore wheelchair users' perspectives on existing VR gaming systems.

\section{Survey Description and Procedure}

The survey asked questions around respondents' experience with gaming and VR systems (or reasons for non-engagement), and their expectations and perspectives thereof. First, respondents provided demographic information including wheelchair use and gaming experience. Then, we explored experience with and perspectives on VR gaming among those who had previously used VR systems. If respondents had no experience with VR, we asked about reasons for non-use, and their expectations of VR. Finally, the survey invited all respondents to describe the kind of VR experiences they would be interested in (if any), explored their perspectives on full-body and wheelchair-controlled VR interaction, and touched upon respondents' perspectives on the representation of wheelchair use within the VR environment (with representation of disability increasingly recognized within the games industry, e.g., [30]). At the end of the survey, all respondents were given the opportunity to give feedback.

\section{Characteristics of Respondents}

The survey received 25 responses from a broad range of participants (average age 33, range 18 to 55; 16 male, 7 female, 2 non-binary). Fourteen respondents used powered wheelchairs, ten participants were manual wheelchair users, and one person reported switching between both options. On average, respondents had been using a wheelchair for 5-10 years (median), ranging from less than a year to more than 35 years of wheelchair use. The majority of respondents reported spending substantial time on games (on average 10-20 hours per week). Platform and genre preferences were varied, ranging from casual games to real-time strategy games, first-person shooting games and role-playing games; many respondents reported playing games online. Nine participants had previously used VR, with systems including lightweight approaches such 
as Google's cardboard VR and more complex solutions such as the Oculus and HTC Vive.

\section{Data Analysis}

Data were analyzed using inductive Thematic Analysis following the protocol outlined by Braun and Clarke [9]. Survey responses were thoroughly read and coded by the main investigator (which is in line with [9] who do not recommend multiple coders for inductive analysis). A total of 116 data points were assigned an initial set of 57 codes which were refined into five final themes, spanning 24 codes.

\section{Results}

We crafted five themes: the Challenging Integration of Embodied Play (Theme 1), the Ambivalent Role of Representation of Mobility Disability (Theme 2), Games as a Means of Escaping Reality (Theme 3), and the Participation in the Brave New World (Theme 4) and Practical Barriers to VR Use (Theme 5).

Theme 1: Challenging Integration of Embodied Play

This theme summarizes respondents' perspectives on full-body movement-based interaction in the context of VR.

Regarding the need for Adaptive and/or alternative control schemes, an overwhelming majority of respondents found fullbody interaction problematic, in some instances also revealing frustration with their own bodies rather than challenging the accessibility of the interaction paradigm, e.g., "It's not so much the control schemes as the fact it's your whole body and part of mine sucks." (P25, manual wheelchair user); "While I enjoy the VR from the perspective of the eyes, the rest of my body does not fit with motion control schemes of VR." (P18, manual wheelchair user). The role of upper body movement in particular was debated, with some participants suggesting it as a solution to accessibility barriers, and others pointing out that it was difficult for them, e.g., "I have some limitations on the use of arms. A lot of games rely on you using hand based motion controls, a lot which I pretty much rule out and instead use a fixed place game controller." (P18, manual wheelchair user). Likewise, participants reflected on difficulties when having to engage in repetitive motion, and many commented on the weight of VR controllers and their combination with wheelchairs.

We could not identify differences between manual and powered wheelchair users, suggesting that within both groups, preferences and needs are varied. Generally, respondents saw wheelchair input as an opportunity, expressing interest in a control scheme that would integrate wheelchairs in full-body interaction, e.g., one participant commenting that "Its a pretty amazing idea. I often forget the wheelchair is there, for it to become part of the gaming control scheme is exciting." (P18, manual wheelchair user). However, there were a number of concerns about feasibility and safety of embodied play, with respondents commenting on space limitations in their own home, and pointing out potential instances of vulnerability relating to embodied play and mobility disability, e.g., "Having some enemy rushing right up to me, especially on my 'bad side' is incredibly, incredibly stressful and even scary - I might end up backing up so quickly or rushing away that I hit a wall or person or something." (P25, manual wheelchair user).
Theme 2: Ambivalent Role of Representation of Mobility Disability

Our results show that representation of mobility disability in games, e.g., including an avatar who has a visible disability, is a far-reaching design decision that engages some players and discourages others. The large majority of respondents expressed some interest in representation of disability, suggesting it would be valuable as an optional feature, but highlighted that "[...] it would depend on the game" (P17, manual wheelchair user). Additionally, respondents highlighted the value of diverse representation extending beyond wheelchairs as a means of educating non-disabled players, e.g., "Sure, but I'd also like to see walkers, canes of various types, etc. I find a lot of times people think about including physically disabled characters, it's wheelchairs only. And people tend to think if you can walk at all and then use aides, especially a wheelchair, you're just 'faking' or something. Nope, couldn't possibly be a range between total immobility and able to run marathons..." (P25, manual wheelchair user). In terms of negative perspectives on representation of mobility disability, participants' perspectives were strongly linked with the following theme, suggesting that one of their motives to engage in play is escapism: "[...] people who use wheelchairs usually like the idea of being characters that don't use them. We aren't judged by others - we are all the same in video games." (P10, powered wheelchair user).

\section{Theme 3: Games and VR as a Means of Escaping Reality}

In terms of immersing oneself in virtual worlds, participant responses suggest that immersion is one of the most important elements that contributes to enjoyment of VR gaming, and also one of the things respondents who had not used VR before were hoping to get out of the experience. This expectation was strongly linked with the reflections on being a person without a disability, with a substantial number of respondents regarding games and VR as an opportunity "to act beyond my physical capabilities" (P3, manual wheelchair user) and that "VR gives you the ability to be something/someone your not or to do something you wouldn't normally be able to do [...]." (P17, manual wheelchair user). However, they also recognized potential dangers of the fidelity of VR, e.g., one participant commented that "I've always used computer games as means of managing pain and having experiences I cannot physically do (running, jumping, swimming). [...] But the sense of realism also has side effects, wanting more to do the things you cannot do (since it becomes closer to reality) rather than controlling an avatar where its very clearly fantasy." (P18, manual wheelchair users), suggesting that there might be harm in virtually exploring experiences that are inaccessible in the real world. Some respondents expressed concern about the implications of wheelchair input for their player experience, suggesting that "It might bring me back into the 'real world' a little bit too much, [...]." (P9, powered wheelchair user).

\section{Theme 4: Participation in the Brave New World}

This theme summarizes the general excitement that respondents expressed about the potential of VR, with the large majority being positive about the potential that the technology could offer. Those who had previously used VR in particular appreciated the new technology, e.g., "I've only experienced it once 
but it was pretty amazing." (P22, manual wheelchair user) and another stating that "It's pretty awesome but not ideal for someone sitting down" (P17, manual wheelchair user). This hints at a growing challenge associated with complex novel entertainment technologies: a number of participants expressed frustration about the large technical advancement that comes with VR and full-body interaction, which at the same time introduces a range of limitations that results in people with mobility disabilities being excluded from immersive, realistic play, e.g., "Feels like the movement to a more real experience in terms of control schemes removes my chance to participate in such games." (P18, manual wheelchair user), and another one commenting "[Accessibility] has been my biggest concerns in VR. People with certain disabilities will be left behind during the video game revolution." (P10, powered wheelchair user), highlighting the need for further accessibility efforts in this area.

\section{Theme 5: Practical Barriers to VR Use}

The final theme summarizes Practical Barriers to the use of VR that we encountered during analysis. In terms of game accessibility, there were some instances that suggest that the complexity of VR systems (i.e., a fairly heavy, head-worn device with wiring) introduced a new accessibility challenge, e.g., "Looks too heavy to sit in my head for too long." (P9, powered wheelchair user). Furthermore, one participant pointed out that the current generation of VR headsets was incompatible with other assistive technology they depended on, "I tried to straps did not play nice with my CPAP mask though." (P8, powered wheelchair user), "Dealing with a cable/umbilical while moving/turning a chair is difficult." (P21, manual wheelchair user). In terms of non-engagement with VR, the "lack of opportunities to test $V R^{\prime \prime}$ in combination with the financial cost of current VR systems was brought up by the majority of respondents. While the financial issue is of course a general barrier to VR use, some respondents pointed out that disability amplified the issue, e.g., the price "is too high to buy to try to see if it will work" (Pl, powered wheelchair user).

\section{Implications for Design}

Here, we summarize our findings and refine the results into initial implications for the design of VR games that will guide our design process throughout the project.

Offer flexible movement-based control schemes, and implement sedentary input as fallback option. Our findings strongly emphasize the need for flexible control schemes when working with movement-based input. Different degrees of disability (e.g., paraplegia vs. tetraplegia) have implications for the general design of movement-based interaction for players, i.e., whether games can leverage arm movement, or games should rely on head or finger movement only, calling for vastly different control schemes for different groups of wheelchair users. Additionally, it is important to recognize different expressions of mobility disability. For example, some players might want to control a game with the stronger side of their body, might be interested in the optional inclusion of legs, or would need an adapted pacing of play to allow for more extensive periods of relaxation where game control does not require substantial movement.
Consider characteristics of the wheelchair when designing interaction paradigms. Using a wheelchair while engaging with VR has implications on multiple levels: First, designers need to be aware of practical issues when expecting players to use hand-held controllers while also moving the wheelchair, and challenges regarding cable management when using wired headsets. Second, it is important to understand the increased space requirement for extensive wheelchair movement and players' concerns of hitting walls and/or furniture while being immersed in VR. Therefore, depending on VR hardware and deployment setting, games for wheelchair users should either integrate hands-free full-body movement-based control schemes, or exclusively focus on controller-based controls while also taking into account range of motion of intended target audiences. In the case of wheelchair movement-based schemes, excessive turning should be avoided, and movement should be limited to a small physical area.

Avoid mandatory representation of disability. Our findings show that representation of disability particularly by means of including a player avatar with a mobility disability is a controversial feature, with a substantial group of players wanting to engross themselves in play as non-disabled characters, while others anticipating benefits of representation. Therefore, we strongly recommend to include representation of disability as an optional feature, or opting for game implementations that leave an avatar's physical abilities up to the imagination of the player (e.g., by choosing a first-person perspective). When opting for representation, designers should consider mobility disabilities beyond wheelchair use.

\section{PART 2: DESIGNING WHEELCHAIR-ACCESSIBLE VR} GAMING - EXPERIENCE PROTOTYPES

Drawing from the initial survey, we present case studies of three experience prototypes that we developed to study how wheelchair users engage with VR games: Karamaisu Slope, Dungeon Spell and Space Travel (see Figure ??). Here, we give an overview of the design rationale, technical implementation, and integration of wheelchair controls. Our design process used our survey results to identify key accessibility challenges to be considered in our game designs, and also leveraged these initial findings to gain insights into player preferences in terms of game genres and themes. We further draw from insights on game design for wheelchair users [14]. In our work, we treat the case studies as experience prototypes [32] to derive further insights into the implications of wheelchair use for engagement with VR games. Both games were implemented using Unity3D, which allows straightforward deployment on VR platforms. In terms of VR hardware, we work with the HTC Vive headset, which provides a highresolution display and tracking of head position and pose. To track the player's body and wheelchair movement within the VR environment, we leverage the Kinect v2 depth camera, which we integrate with Unity. Wheelchair movement is inferred from body movement in a similar way as suggested by the KinectWHEELS toolkit [16].

\section{Case Study 1: Karamaisu Slope}

Karamaisu Slope (KS) is a skiing game in which players steer down a hill while trying to successfully pass through as many 


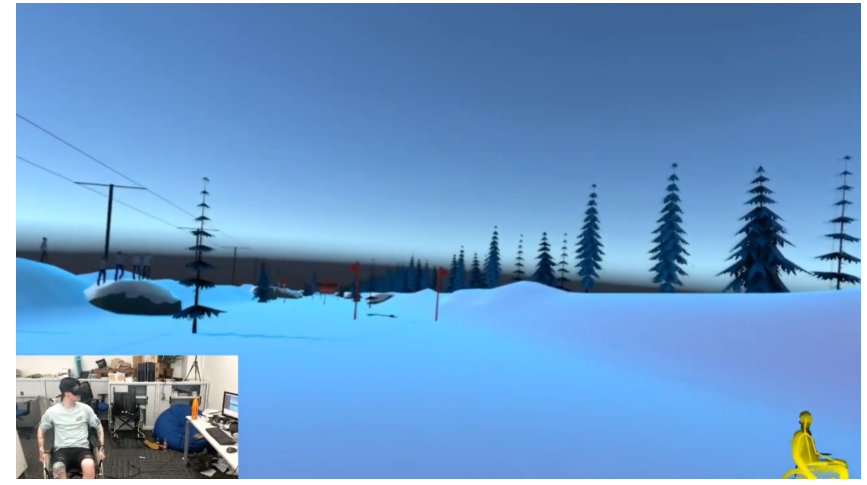

Figure 1. Screenshot of Karamaisu Slope.

slalom gates as possible, as quickly as they can (see Figure 1). The game theme was chosen based on previous work [14] and survey findings suggesting that some players would like to explore activities that are difficult to access in the real world. Players guide a skiing avatar between slalom posts by controlling speed and turning left and right. While passing the posts is not required, successfully doing so provides players with a time bonus. The course is filled with obstacles such as trees and rocks, which have to be additionally navigated. Additionally, the game would assist players with their movement if they are moving away from the slalom gates, it will gently apply a force to move them back to the correct path. It takes about 1:30 minutes to complete an episode. Player Interaction. As input, players raise or lower their arms to control speed of their avatar. Players further need to rotate their wheelchair to the direction they want the avatar to move in.

\section{Case Study 2: Dungeon Spell}

Dungeon Spell (DS) is a fantasy-themed dungeon crawler in which players defend themselves against an onslaught of fantastical enemies (see Figure 2). The game is played from a first-person perspective, and players automatically move along a preset trajectory (in a similar fashion as employed in rail shooters), firing fireballs to defeat oncoming enemies. The player avatar can be hit by the enemies, and respawns after a short break if the player's health value reaches a minimum. Progressing through the entire dungeon, including the final boss battle, takes about three minutes. Player Interaction. Avatar movement is automated and does not require player input. To engage in combat, the player releases a fireball by performing a punching gesture with one arm. Automation was introduced to reflect survey findings where respondents suggested that neither body-based nor controller-based input would be feasible for them at scale.

\section{Case Study 3: Space Travel}

Space Travel (ST) is an endless runner game in which players control a spaceship (see Figure 3). The aim of the game is to score points by navigating through goal hoops, whilst avoiding obstacles such as asteroids. The game ends either when the spaceship is damaged by the asteroids, or when the time limit of two minutes is reached. Player Interaction. Players move their head orientation to direct the trajectory of their ship, and optional single-arm input (punching) to

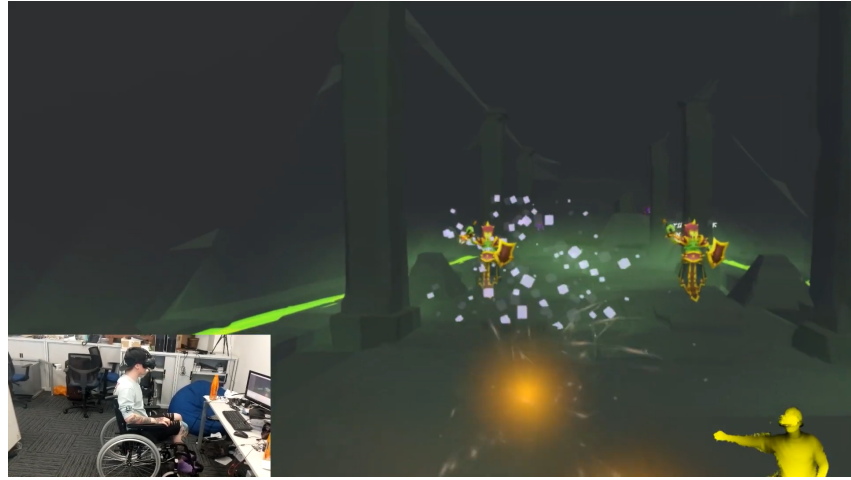

Figure 2. Screenshot of Dungeon Spell.

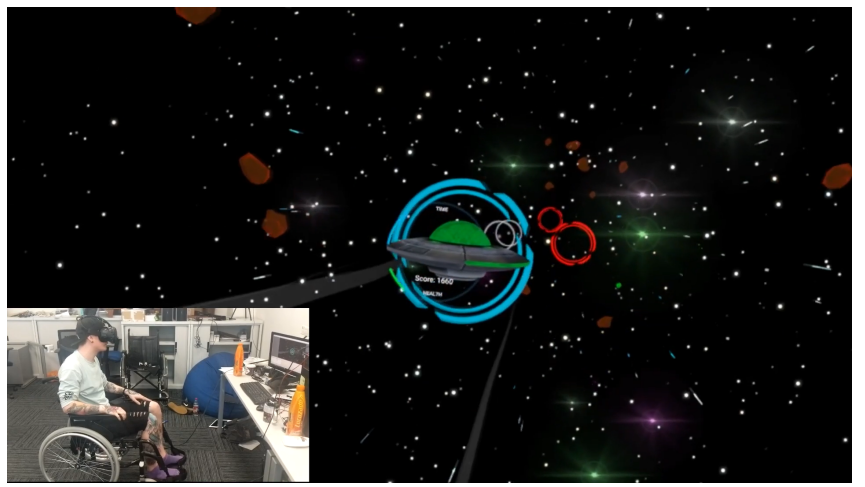

Figure 3. Screenshot of Space Travel.

engage in combat. If players do not make arm input, combat is triggered automatically. We took this design decision to make the game accessible to players who found head movement most comfortable, reflecting survey findings.

\section{PART 3: EVALUATION}

The evaluation was carried out in two steps: First, we worked with WheelPower to carry out an evaluation of accessibility, usability and player experience in the context of a major sports event for wheelchair users, and also recruited participants at Kidz to Adultz Middle, a major exhibition targeting young people with disabilities in the UK. Second, we worked with the Back-Up Trust to carry out an in-depth qualitative exploration of the VR games. While carrying out the evaluation in conjunction with public events and/or at the premises of third parties was a trade-off in terms of being able to control the experimental environment, we decided to take this step to increase accessibility of the activity: from past experience, we knew that travel to university campuses to participate in lab evaluations can be a barrier for wheelchair users. Applying a quantitative approach in Study 1 allows us to screen the broader experience provided by the games along with accessibility and usability concerns, while applying a qualitative, small-scale approach in Study 2 provides in-depth insights into the detailed experience of players through case studies. 


\section{Study 1: Usability, Accessibility and PX}

We explored two research questions (RQs): are the VR games accessible and usable for wheelchair users (RQ1), and do they provide a positive player experience (RQ2)?

\section{Measures}

Because this study was run in conjunction with public events and we wanted to reduce participant burden in terms of duration of the study, we adapted two existing questionnaires into a shorter format to be administered through structured interviews: The first set of questions was selected to represent the main dimensions of the Player Experience and Need Satisfaction (PENS) questionnaire [28], exploring the dimensions of competence, autonomy, relatedness, presence, and ease of use; in our work we focus on the subscales of competence, autonomy, presence, and ease of use. The second set of questions was selected in line with the NASA-TLX [19], a tool to assess task load, including items investigating mental demand, physical demand, and temporal demand when engaging with technology. In the interview, participants were first asked to respond to each question using a three-point scale, and then given opportunity to elaborate.

\section{Participants and Procedure}

We recruited 14 wheelchair users (average age 34, range 1956; 2 female, 1 non-binary) to participate in the study. All participants were active wheelchair users (duration of use ranging from two months to 23 years; 12 manual wheelchair users). Most participants had played games before (preferences ranging from casual games to FPS and RTS); 12 were experienced with motion-based controls (predominantly having used the Nintendo Wii); eight participants had used VR systems before (often at work or to watch movies). None of the participants were active VR gamers.

Participants took part in the study at a wheelchair event hosted by WheelPower, a charity that focuses on providing opportunities for disabled people to get into sports and lead active lives. Firstly, participants were briefed with information about the games and the study and asked to provide consent. Participants were free to choose any of the three games they wanted to try after each game being described to them; we took this step to ensure we would not expose vulnerability of participants who would prefer to not engage with a sport such as skiing (see survey findings regarding the exploration of inaccessible activities). An individual game session lasted around 3 minutes; some participants played all three games which took around 15-20 minutes in total. In terms of absolute numbers, 13 participants played KS, 9 participants played ST, but only 3 participants engaged with DS which we therefore exclude from quantitative results (but refer to in qualitative findings).

All participants took part in a semi-structured interview which included demographic information and explored their experience with the prototypes. At the end of the interviews, participants were thanked for their time and given time to ask questions. The research protocol was approved by the ethics board at the University of Lincoln.
Table 1. Average scores (Mean, Standard Deviation) for PENS and TLX (3-point scale, 3 = agree).

\begin{tabular}{llllll} 
& & \multicolumn{2}{c}{ KS } & \multicolumn{2}{c}{ ST } \\
\hline \multirow{3}{*}{ PENS } & Competence & 2.55 & 0.51 & 3.00 & 0.00 \\
& Autonomy & 2.92 & 0.28 & 3.00 & 0.00 \\
& Presence & 3.00 & 0.00 & 3.00 & 0.00 \\
\multirow{5}{*}{ TLX } & Ease of Use & 2.75 & 0.54 & 2.93 & 0.27 \\
& Physical Demand & 1.25 & 0.71 & 1.00 & 0.00 \\
& Mental Demand & 1.87 & 0.73 & 2.00 & 1.00 \\
& Temporal Demand & 3.00 & 0.00 & 2.85 & 0.38 \\
\hline
\end{tabular}

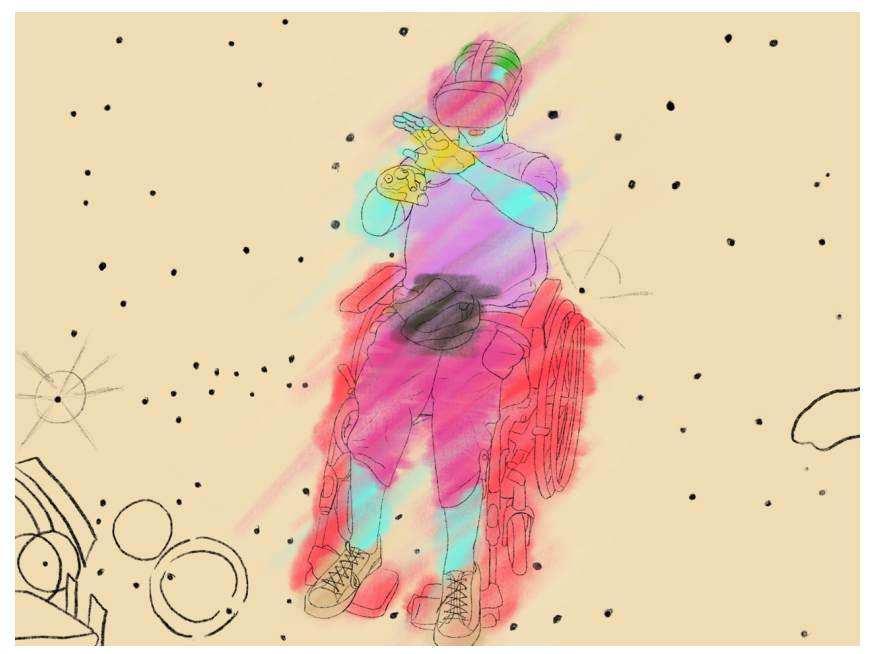

Figure 4. Sketch of a player engaging with Space Travel.

\section{Results}

We structure our results around quantitative data and use qualitative data to illustrate findings. We report our results following the research questions, drawing from quantitative and qualitative participant feedback.

RQ1: Are the VR games accessible and usable for wheelchair users? On a basic level, the VR setup and games were accessible and usable for study participants as reflected by quantitative findings (adapted versions of the PENS and NASA-TLX; see Table 1 for results for KS and ST; DS excluded due to small $\mathrm{N}$ ) that show a relatively low physical demand (although high temporal demand likely induced by the nature of games) and positive player experience including high ease of use. In terms of usability of the VR hardware, $13 / 14$ participants reported that it was comfortable to wear, with one participant noting they would not be able to wear it "for more than 20 minutes" (P11). Likewise, there was only one instance where a participant had to readjust the wires of the headset; two participants explicitly pointed out that the control scheme helped avoid entanglement. In terms of feeling safe while immersed in VR, participants were aware of the risk of collision (reflecting survey findings) but it did not hamper their experience under the given circumstances; with one participant pointing out that it was "not stressful and part of the fun as long as you don't break anything" $(P 7)$, and others commenting that being watched by others while playing helped them feel comfortable. In terms of the 
control schemes (head control vs. wheelchair and gesture controls), participants were very positive about the inclusion of the wheelchair in interaction, with one participant pointing out that "that you could move your wheelchair that was unbelievable, I obviously loved that." (P6). In particular, participants were excited about its contribution to immersion and realism, and accessibility implications. In contrast, head controls were perceived as limiting; here, participants suggested a bigger input alphabet while also recognizing the benefit of this scheme for players with no upper-body control. Considering the integration of hand and arm gestures, 4/14 participants reported issues, e.g., "[My] arms felt tired because of shoulder problems. " (P1O), highlighting the need to explore adaptive control schemes in more detail. Finally, the biggest point of concern was the level of control over movement in the virtual world ( $7 / 14$ participants). Here, some participants commented that there were instances in which the tracking system was not responsive enough on a technical level; additionally, participants did not appreciate automation (e.g., movement on rails in Dungeon Spell), e.g., one participant commented that "[...] you weren't in control of the movement." (P14).

RQ2: Do the games provide a positive player experience? In general, quantitative findings show that player experience with KS and ST was positive (see Table 1; DS not included in analysis due to small N). Qualitative results support this notion, but draw a more nuanced picture of player preferences: players engaged with Karamaisu Slope and Space Travel more frequently and for longer periods than with Dungeon Spell. Regarding elements that contributed to a positive player experience, $8 / 14$ participants commented on feeling immersed in the virtual world; one participant however pointing out that the experience was overwhelming. Along these lines, 6/14 participants pointed out that they appreciated realism of the skiing game in particular, e.g., "It was nice to be back on the skis, I was a keen skier so ye, very realistic." (P4). Here, survey findings were mirrored in the sense that participants appreciated the opportunity to gain access to an inaccessible activity, e.g., "[ the ski game was interesting because it] relates to what I used to be able to $\mathrm{do}^{\prime \prime}(\mathrm{Pl})$. In terms of areas for improvement and desirable features, $5 / 14$ participants would have liked to be more challenged by the games (with one however feeling overwhelmed), and suggested to increase game speed (particularly downhill skiing speed) and further supporting player autonomy through more varied input options. In terms of specific features, inclusion of additional non-player characters and multiplayer options were suggested.

\section{Changes to the Experience Prototypes}

Based on these findings, we further refined the three games: most importantly, we updated the gesture recognition mechanism to account for variances in player movement. Adapting the gestures to personal abilities allows players to customise the gesture they would do; for example, a player can choose to do a full punch (i.e., fully extend their arm in front of them) or to wave their hand slightly to achieve the same action. All three games also received further polish in their presentation: fellow skiers (non-player characters) were added to Karamaisu Slope. The game design of Space Travel changed slightly to allow the player to shoot at hazards, using the same punching gesture setup as in Dungeon Spell. This offers more choice in terms of avoiding or destroying hazards. In Dungeon Spell, enemy placement was changed to allow for a linear ramp in difficulty, and the cool-down on how often the player can fire was reduced. One element that we did not change despite participants' criticism was the degree of movement automation in Dungeon Spell, with the goal of studying the impact of this approach on player experience (PX) in more depth in the following step of our research.

\section{Study 2: In-depth Exploration of Second Iteration of Re- search Games}

In the second study, we explored the second iteration of the research games together with four participants who were also experienced wheelchair users.

\section{Participants and Procedure}

Four participants were part of the second study: $\mathrm{Sam}^{2}$ is a 27 year old male who has used a manual wheelchair since a cycling accident eleven years ago. He used to be an active gamer with a strong interest in sports games (e.g., racing games such as Need for Speed series). Sam is an avid skier. Sarah is a 33 year old woman who has been using a manual wheelchair for 23 years. She does not consider herself a 'gamer', but has played games on the Nintendo Wii and Sony Playstation before. James is 45 years old and has used a wheelchair for 27 years. While he typically opts for manual propulsion, he sometimes uses a clip-on motor for support. He regularly plays games (most recently the battle royale game PlayerUnknown's Battlegrounds), and has previously used Samsung's VR headset. Finally, Richard is a 48 years old male who uses a manual wheelchair due to spinal cord injury. He does not play video games because he has no movement in his fingers; he has used phone-based cardboard VR headsets before and is interested in games as a way of connecting with his teenage son. He is an active player of competitive wheelchair rugby.

The study took place at the premises of the Back-Up Trust, a national charity supporting individuals with spinal cord injury. First, participants were given information about the study and asked to provide consent. Then, they were introduced to the games and given time to familiarise themselves with the controls. Next, they were invited to play the games for about 30 minutes. Afterwards, participants took part in open-ended interviews exploring their experience in the light of their individual backgrounds, generally addressing accessibility, usability and player experience (mirroring the areas of interest of study 1), with a set of questions particularly focusing on body-based input and automation. At the end of the interviews, participants were given room to ask questions, and thanked for their time. All interviews were audio recorded; the research protocol was approved by the University of Lincoln ethics board.

\footnotetext{
${ }^{2}$ This and the following names are edited for anonymity.
} 


\section{Data Analysis}

We applied inductive Thematic Analysis (TA) following the research process laid out by Braun and Clarke [9]. All data were read and coded by one researcher; themes that were crafted were discussed and revised with two other members of the research team. This approach is in line with [10]; please note that inductive TA is a subjective research approach that requires in-depth engagement of a single individual with the data. In total, we coded 283 data points and crafted four themes.

\section{Results}

We crafted four themes from the data: Personalizable and adaptive embodied play (Theme 1), Matching player expectations regarding autonomy and control (Theme 2), Physical risk as source of enjoyment or worry (Theme 3), and Relevance of player types (Theme 4).

Theme 1: Personalizable and adaptive embodied play. This very prominent theme addresses the need for personalizable and adaptable movement-based controls that allow wheelchair users to tailor VR experiences. Although our sample exclusively consisted of manual wheelchair users who had a spinal cord injury with a high degree of wheelchair control, physical abilities were broad: For example, Sam was enthusiastic about full-body controls ("I thought it was awesome, [...], you really feel like you're kind of immersed in that world!"), whereas Sarah was interested in traditional, controller-based input. Likewise, Sam reported difficulty with input requiring fine motor control (e.g., using game controllers or hand gestures). There was consensus that adaptability is a basic requirement when aiming to engage wheelchair users in VR. Additionally, participants saw adaptation of controls as a means of increasing health and safety where needed (e.g., avoiding extensive movement when playing on their own) and a way of fitting VR systems into their own homes (which was a point of concern along with cost of VR systems). Generally, results suggest that participants would like to be able to make more drastic adaptations than those currently offered by the games, supporting the initial guideline of offering a broad range of input modalities for VR systems wishing to be accessible for wheelchair users.

Theme 2: Matching player expectations regarding autonomy and control. This theme captures participants' expectations in terms of realism of interaction paradigms and the impact of automation on player experience. Most importantly, a strong preference for realistic mappings of controls (i.e., a match between real-world actions and gestures/movements required to play the games) was expressed particularly for Karamaisu Slope. Here, participants who had skiing experience were most critical of the implementation, with Sam pointing out that "[...] the way that you steer when you sit-ski is quite different.[...] Erm because you basically, initiate the turn using your wrist almost." and continuing to make suggestions to improve the realism of the control scheme. Interestingly, Space Travel was broadly appreciated by participants while only offering a restricted yet responsive input alphabet and simplistic gameplay, whereas Dungeon
Spell offered more complex gameplay while parts of player movement were automated. Mirroring findings from the first study, participants expressed a strong dislike of this design element because it limited opportunity for exploration. Additionally, it reduced control over the fighting mechanic: "I think it can be a bit frustrating [...] because obviously it was too far away and I was punching away with both hands but there was no bullets coming out" (James).

Theme 3: Physical risk as source of enjoyment or worry. Our data supports the notion that physical risk associated with movement-based play can serve as a source of enjoyment or worry. While Sarah and Sam were concerned about immersing in VR, participating in extensive movement, and experienced displacement, Richard experienced this process as a thrill that contributed to his enjoyment of the VR experience, a perspective mirrored in his participation in wheelchair rugby, a sport with significant risk of physical injury. This draws interesting links with previous work on movement-based play $[23,22]$ which highlights that physical risk can be employed as a design strategy. However, in the context of VR there is a clear trade-off between health and safety and the experience, where Sarah suggested that she would be more comfortable with small-scale movement not involving the wheelchair, an aspect dismissed by Richard: "Umm, that would take away something from the game though wouldn't it. It's a delicate balance." This highlights a challenge in the design of VR games: we must embrace all properties of this technology, but make careful choices to ensure reasonable safety. Here, awareness of individual preferences: regardless of physical abilities, players may be attracted to (or discouraged by) experiences that pose physical risk, so VR systems need to be crafted accordingly.

Theme 4: Relevance of player types. We crafted this theme to represent different perspectives on the nature of play that we encountered in our data. Linked with the immersive nature of VR gaming, we observed two main dimensions that seemed to affect players' experiences: while some participants appreciated the competitive nature of play, and were particularly attracted to Karamaisu Slope as a game that offers clear goals and performance indicators, others highlighted their desire to explore VR games that are free from competition, strongly focusing on curiosity and exploration: "[...] it's a bit more chilled out, you kind of, there's no like kind of pressure you know" (Sam). This observation is linked to the general proposition that there are different player types (e.g., individuals who prefer competition over other features of play [24]). While not uncontested, these types seem relevant for immersive VR gaming; in the context of our work, participants suggested the addition of unstructured modes of play that would allow them to satisfy their curiosity in the same gaming environment that can also be engaged with when striving to fulfil specific goals.

\section{DISCUSSION}

The results of our project show that flexibility and adaptability of interaction paradigms are important to provide accessible and engaging VR games, that wheelchair users are a hetero- 
geneous target audience in terms of preferences and needs, and that representation of disability in VR gaming is an aspect for further exploration. Here, we discuss implications of our findings.

\section{Body-based VR Games for Wheelchair Users}

\section{The Role of Adaptation and Automation}

In response to the need for adaptive and flexible interfaces that emerged from the initial survey, we created games that offer adaptation on two levels: We implement fundamentally different control schemes, provide flexibility within these schemes, and automate interaction to provide more complex gameplay despite a small input alphabet. While both evaluations highlight that full-body play was generally appreciated and contributed to perceived realism and immersion, they also reveal the degree of adaptation needed to accommodate broad groups of wheelchair users. Here, flexible control schemes increase accessibility, but potentially decrease match between realworld and in-game actions, an element that was considered important in games that mimic real-world activities. Regarding automation, results indicate tension between players' desire for autonomy and our designerly attempt to create accessible versions of games with complex gameplay: in Dungeon Spell, automation of player movement was perceived as a limitation of control rather than a supporting feature; however, gentle correction of player movement in Karamaisu Slope went unnoticed. Here, future work should work with wheelchair users to explore benefits and drawbacks of automation in more detail, and identify which elements are suitable for this approach (if at all).

\section{Representation of Disability in Body-based Play}

Immersive body-based play has previously raised questions around representation of disability, and the option for player avatars to reflect disabilities of players [14]. While adequate representation of disability in games is supported by the academic community (e.g., [11]) and industry consultants (e.g., [30]), survey responses draw a more nuanced, if not critical view: integration of disability through the player avatar is perceived as a risk to the value of games as a means of escaping reality; in fact, a number of respondents voiced an interest VR as a means of playfully exploring inaccessible real-world activities. This pushback could be interpreted as internalization of stereotypes and negative views associated with disability [26] (and thus not wanting to be disabled in the virtual world), but could also simply be an expression of wanting to leverage VR to experience being someone else and to enjoy novel experiences, which is one of the core drivers for people to engage with digital games [29].

\section{Revisiting Our Initial Implications for Design}

We initially formulated three implications for design for VR games for wheelchair users: flexible control schemes, accounting for wheelchair characteristics, and avoiding mandatory representation of disability. Our findings generally support the considerations, but provide more nuanced perspectives: control schemes need not only offer flexibility, but also adaptability within a given scheme (e.g., to range of motion of an individual); sedentary input is relevant to reflect a wide range of player preferences. Accounting for wheelchair characteristics is important, however, our findings remind us to focus on the human being occupying the chair: our findings highlight that aspects such as risk-taking and the enjoyment thereof are linked with individual preferences; generally removing all physical risk from games for wheelchair users might therefore be inappropriate. Likewise, close attention needs to be paid to individual abilities, linking back to the theme of flexible controls. Finally, leaving imagining their virtual self up to the player rather than including avatars with disabilities worked well in our research setting, but should be explored in more detail in the future to address the need to refine considerations for the design of representation of disability in VR games.

\section{Reflections on Disability and VR Gaming}

Our work broadly highlights the importance of accessible VR, which is perceived as a futuristic technology that should be available to everyone. In this, there lies a core challenge for the development of ever more immersive and therefore bodycentric systems. Typically, technology is designed for healthy white people, not accounting for minority bodies [5]. In this design perspective lies the risk of leaving behind audiences who see unique benefits in novel technologies: VR gaming offers the opportunity of accessing inaccessible experiences (if players wish to do so), and is seen as a technology that when designed right - has potential to move the person into the foreground and stigma into the background. However, accessibility concerns need to be understood on a broader level than interaction paradigms and gameplay: we need to develop a deeper understanding of how to involve and represent minority bodies in body-based immersive play, and have to situate their perspectives on VR in the broader context of their lived experiences, taking into account perspectives on disability, gaming preferences, and last but not least socioeconomic barriers associated with the use of novel gaming technology that were highlighted in our work.

\section{LIMITATIONS AND FUTURE WORK}

Our work needs to be interpreted in the light of a number of limitations. First, across all parts of our research, we worked with small participant samples which are typical for accessibility work, but limit generalizability. Additionally, the first evaluation was carried out in conjunction with a sports event; therefore, playing sessions and follow-ups with participants were shorter than we would have liked (e.g., only partial use of the PENS questionnaire). Second, both studies involving gameplay only look at single-session engagement of wheelchair users with VR: here, it would be interesting to explore how experiences develop over time. Third, we would like to point out that not all issues raised in the survey and studies can be addressed by game design: for example, the cost of VR and increased space requirements in case of wheelchair use remain challenging. In terms of future work, we believe that participatory rather than user-centered design of wheelchair-accessible VR games is an avenue to further explore automation, along with general research into the representation of mobility disability in VR. 


\section{CONCLUSION}

VR gaming offers the opportunity of crafting novel immersive experiences that engage players. However, little is known about the accessibility for players with disabilities. Our work takes a first step into the direction of understanding how mobility impairment impacts the way wheelchair users engage with and experience VR games. Our work suggests that VR has a large appeal for this audience, but that careful adaptation is required to ensure accessibility of full-body interaction in VR environments, and that considerations for the design of VR games for wheelchair users need to extend to the practical design of such environments, too: to create truly accessible and engaging experiences, we need to better understand the impact of mobility impairment on player experience, and further explore challenges associated with player autonomy within virtual worlds along with the impact that representation of disability has on player experience.

\section{ACKNOWLEDGEMENTS}

We thank all survey and study participants for their time and thoughtful feedback. We would also like to thank WheelPower, the Back-Up Trust and the Belgrade Theatre Coventry for supporting our research, and Leif Walker-Grant for his exploratory research.

\section{REFERENCES}

[1] 2017. Online Survey: People with Disabilities \& VR Accessibility. https:

//disabilityvisibilityproject.com/2017/01/03/vr/. (2017). Accessed: 2018-09-18.

[2] 2019a. Steam. (2019). https://store.steampowered.com Accessed: 2019-09-19.

[3] 2019b. SteamVR. (2019). https://store.steampowered.com/steamvr Accessed: 2019-09-19.

[4] 2019. WalkinVR Driver. (2019). https://www. walkinvrdriver. com/ Accessed: 2019-09-18.

[5] E. Barnes. 2016. The Minority Body: a theory of disability. Oxford University Press, New York.

[6] Jen Beeston, Christopher Power, Paul Cairns, and Mark Barlet. 2018. Accessible Player Experiences (APX): The Players. In Computers Helping People with Special Needs, Klaus Miesenberger and Georgios Kouroupetroglou (Eds.). Springer International Publishing, 245-253.

[7] LouAnne Boyd. 2019. Designing Sensory-Inclusive Virtual Play Spaces for Children. In Proceedings of the 18th ACM International Conference on Interaction Design and Children (IDC '19). ACM, New York, NY, USA, 446-451. DOI :

http://dx.doi.org/10.1145/3311927.3325315

[8] Lal Bozgeyikli, Evren Bozgeyikli, Matthew Clevenger, Andrew Raij, Redwan Alqasemi, Stephen Sundarrao, and Rajiv Dubey. 2015. VR4VR: Vocational
Rehabilitation of Individuals with Disabilities in Immersive Virtual Reality Environments. In Proceedings of the 8th ACM International Conference on PErvasive Technologies Related to Assistive Environments (PETRA '15). ACM, New York, NY, USA, Article 54, 4 pages. DOI : http://dx.doi .org/10.1145/2769493.2769592

[9] Virginia Braun and Victoria Clarke. 2006. Using thematic analysis in psychology. Qualitative Research in Psychology 3, 2 (2006), 77-101. DOI :

http://dx.doi.org/10.1191/1478088706qp063oa

[10] Virginia Braun and Victoria Clarke. 2013. Successful Qualitative Research: A Practical Guide for Beginners. SAGE.

[11] D. Carr. 2014. Ability, Disability and Dead Space. Game Studies 14, 2 (2014).

[12] Stephen Cuzzort and Thad Starner. 2008. AstroWheelie: A Wheelchair Based Exercise Game. In Proceedings of the 2008 12th IEEE International Symposium on Wearable Computers (ISWC '08). IEEE Computer Society, Washington, DC, USA, 113-114. DOI : http://dx.doi.org/10.1109/ISWC. 2008.4911599

[13] Shirley G. Fitzgerald, Rory A. Cooper, Emily Zipfel, Donald M. Spaeth, Jeremy Puhlman, Annmarie Kelleher, Rosemarie Cooper, and Songfeng Guo. 2006. The development and preliminary evaluation of a training device for wheelchair users: The GAMEWheels system. Disability and Rehabilitation: Assistive Technology 1, 1-2 (2006), 129-139. DOI : http://dx. doi .org/10.1080/09638280500167639

[14] Kathrin Gerling, Kieran Hicks, Michael Kalyn, Adam Evans, and Conor Linehan. 2016. Designing Movement-based Play With Young People Using Powered Wheelchairs. In Proceedings of the 2016 CHI Conference on Human Factors in Computing Systems (CHI '16). ACM, New York, NY, USA, 4447-4458. DOI : http://dx.doi.org/10.1145/2858036.2858070

[15] Kathrin Gerling, Michael R. Kalyn, and Regan L. Mandryk. 2013a. KINECTwheels:

Wheelchair-accessible Motion-based Game Interaction. In CHI'13 Extended Abstracts on Human Factors in Computing Systems (CHI EA '13). ACM, New York, NY, USA, 3055-3058. DOI :

http://dx.doi.org/10.1145/2468356.2479609

[16] Kathrin M. Gerling, Regan L. Mandryk, and Michael R. Kalyn. 2013b. Wheelchair-based Game Design for Older Adults. In Proceedings of the 15th International ACM SIGACCESS Conference on Computers and Accessibility (ASSETS '13). ACM, New York, NY, USA, 27:1-27:8. DOI : http://dx.doi .org/10.1145/2513383.2513436

[17] Hannah Gillis and Ben Peck. 2017. Sources of Inspiration for Approaching Accessibility in VR. (2017). https://www.gdcvault.com/play/1024618/ Sources-of-Inspiration-for-Approaching Accessed: 2018-09-18. 
[18] Roland Graf, Sun Young Park, Emma Shpiz, and Hun Seok Kim. 2019. iGYM: A Wheelchair-Accessible Interactive Floor Projection System for Co-located Physical Play. In Extended Abstracts of the 2019 CHI Conference on Human Factors in Computing Systems (CHI EA '19). ACM, New York, NY, USA. DOI : http://dx.doi.org/10.1145/3290607.3312792

[19] Sandra G Hart and Lowell E Staveland. 1988. Development of NASA-TLX (Task Load Index): Results of empirical and theoretical research. In Advances in psychology. Vol. 52. Elsevier, 139-183.

[20] N. W. John, S. R. Pop, T. W. Day, P. D. Ritsos, and C. J. Headleand. 2018. The Implementation and Validation of a Virtual Environment for Training Powered Wheelchair Manoeuvres. IEEE Transactions on Visualization and Computer Graphics 24, 5 (May 2018), 1867-1878. DOI : http://dx.doi.org/10.1109/TVCG.2017.2700273

[21] Lange B George S Deutsch JE Saposnik G Laver, KE and M Crotty. 2017. Virtual reality for stroke rehabilitation. Cochrane Database of Systematic Reviews 11 (2017). DOI :

http://dx.doi.org/10.1002/14651858.CD008349.pub4

[22] Joe Marshall, Conor Linehan, and Adrian Hazzard. 2016. Designing Brutal Multiplayer Video Games. In Proceedings of the 2016 CHI Conference on Human Factors in Computing Systems (CHI'16). ACM, New York, NY, USA, 2669-2680. DOI : http://dx.doi.org/10.1145/2858036.2858080

[23] Florian Mueller and Katherine Isbister. 2014. Movement-based Game Guidelines. In Proceedings of the SIGCHI Conference on Human Factors in Computing Systems (CHI '14). ACM, New York, NY, USA, 2191-2200. DOI : http://dx.doi.org/10.1145/2556288.2557163

[24] Lennart E. Nacke, Chris Bateman, and Regan L. Mandryk. 2011. BrainHex: Preliminary Results from a Neurobiological Gamer Typology Survey. In Entertainment Computing - ICEC 2011, Junia Coutinho Anacleto, Sidney Fels, Nicholas Graham, Bill Kapralos, Magy Saif El-Nasr, and Kevin Stanley (Eds.). Springer Berlin Heidelberg, Berlin, Heidelberg, 288-293.

[25] Thomas J. O’Connor, Shirley G. Fitzgerald, Rory A. Cooper, Tricia A. Thorman, and Michael L. Boninger. 2002. Kinetic and physiological analysis of the GAMEWheels system. Journal of Rehabilitation Research and Development 39, 6 (November 2002), 627-634.
[26] Donna Reeve. 2002. Negotiating Psycho-emotional Dimensions of Disability and their Influence on Identity Constructions. Disability \& Society 17, 5 (2002), 493-508. DOI :

http://dx.doi.org/10.1080/09687590220148487

[27] A.A. Rizzo, J.G. Buckwalter, T. Bowerly, C. Van Der Zaag, L. Humphrey, U. Neumann, C. Chua, C. Kyriakakis, A. Van Rooyen, and D. Sisemore. 2000. The Virtual Classroom: A Virtual Reality Environment for the Assessment and Rehabilitation of Attention Deficits. CyberPsychology \& Behavior 3, 3 (2000), 483-499.

DOI : http://dx.doi.org/10.1089/10949310050078940

[28] Richard M Ryan, C Scott Rigby, and Andrew Przybylski. 2006. The motivational pull of video games: A self-determination theory approach. Motivation and emotion 30, 4 (2006), 344-360.

[29] Dimitri Schuurman, Katrien De Moor, Lieven De Marez, and Jan Van Looy. 2008. Fanboys, Competers, Escapists and Time-killers: A Typology Based on Gamers' Motivations for Playing Video Games. In Proceedings of the 3 rd International Conference on Digital Interactive Media in Entertainment and Arts (DIMEA '08). ACM, New York, NY, USA, 46-50. DOI : http://dx.doi.org/10.1145/1413634.1413647

[30] Cherry Thompson. 2019. You Can Take an Arrow to the Knee and Still Be an Adventurer. (2019). https://www . gdcvault . com/browse/gdc-19/play/1026442 Accessed: 2018-09-19.

[31] Ryan Wedoff, Lindsay Ball, Amelia Wang, Yi Xuan Khoo, Lauren Lieberman, and Kyle Rector. 2019. Virtual Showdown: An Accessible Virtual Reality Game with Scaffolds for Youth with Visual Impairments. In Proceedings of the 2019 CHI Conference on Human Factors in Computing Systems (CHI'19). ACM, New York, NY, USA, 141:1-141:15. DOI : http://dx.doi.org/10.1145/3290605.3300371

[32] Peter Wright and John McCarthy. 2010. Experience-Centered Design: Designers, Users, and Communities in Dialogue. Morgan and Claypool Publishers.

[33] Bei Yuan, Eelke Folmer, and Frederick C. Harris. 2011. Game accessibility: a survey. Universal Access in the Information Society 10, 1 (Mar 2011), 81-100. DOI : http://dx.doi .org/10.1007/s10209-010-0189-5 